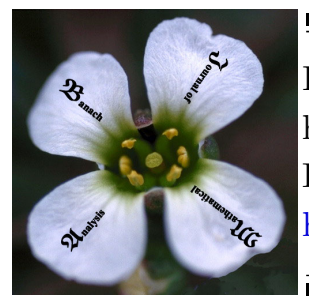

Banach J. Math. Anal. 9 (2015), no. 1, 235-242

http://doi.org/10.15352/bjma/09-1-17

ISSN: $1735-8787$ (electronic)

http://projecteuclid.org/bjma

\title{
ON AN ANALOGUE OF $A B A$ WHEN THE OPERATOR VARIABLES $A$ AND $B$ ARE CONVEX FUNCTIONALS
}

\author{
MUSTAPHA RAÏSSOULI \\ Communicated by Zs. Pales
}

\begin{abstract}
The operator-product $A B A$ appears in many mathematical contexts, such as in algebraic Riccati equation, in operator entropy and in operatormean theory. The purpose of the present paper is to investigate a reasonable analogue of $A B A$ when the positive linear operators $A$ and $B$ are convex functionals. As consequence, the square of a convex functional extending $A^{2}$ is provided as well.
\end{abstract}

\section{INTRODUCTION}

For over the last years, an enormous amount of effort by some authors has been devoted to understand the extension of various operator concepts when the involved operators are replaced by convex functionals. For instance, some operator means, the operator inverse $A \longmapsto A^{-1}$, the operator logarithm $A \longmapsto$ $\log A$, the power operator $A \longmapsto A^{m}(-1<m<1)$, the (Tsallis) operator entropy and the shorted operator have been extended from the case that the variables are positive linear operators to the case that the variables are convex functionals, see $[1,2,3,4,6]$ and the related references cited therein. The above extensions were investigated in the sense that, if $A \longmapsto \phi(A)$ is a map between positive operators then its extension $f \longmapsto \Phi(f)$ for convex functionals satisfies

$$
\Phi\left(f_{A}\right)=f_{\phi(A)},
$$

Date: Received: Jan. 20, 2014; Accepted: Apr. 28, 2014.

2010 Mathematics Subject Classification. Primary 46N10; Secondary 46A20, 47N10, 39B62, $52 \mathrm{~A} 41$.

Key words and phrases. Positive linear operator, generalized inner product, Fenchel duality, point-wise convexity, functional inequalities. 
where $f_{A}$ denotes the quadratic convex functional generated by the positive operator $A$ acting on a Hilbert space $H$, i.e. $f_{A}(u)=(1 / 2)\langle A u, u\rangle$ for every $u \in H$. Further, throughout the study of the above functional extensions it could be observed that the involved topology, used in the related topological properties, is that of point-wise convergence, i.e.

$$
f_{n} \longrightarrow f \text { if and only if } f_{n}(u) \longrightarrow f(u) \text { for all } u \in H
$$

while the used topology for the operator version is the quadratical topology, i.e.

$$
A_{n} \longrightarrow A \text { if and only if }\left\langle A_{n} u, u\right\rangle \longrightarrow\langle A u, u\rangle \text { for all } u \in H \text {. }
$$

As well-known, if an operator sequence $\left(A_{n}\right)$ is such that $A_{n} \geq A$ for all $n$ then, $\left(A_{n}\right)_{n}$ converges to $A$ in norm if and only if $\left(A_{n}\right)$ converges quadratically to $A$.

However, as far as we know, extensions of $A \longmapsto A^{m}$ for $m>1$ as well as that of $A \longmapsto \exp A$, from operators to convex functionals, are not done yet. In particular, a reasonable analogue of $A \longmapsto A^{1 / 2}$ from positive operator to convex functional is well-known [1] while that of $A \longmapsto A^{2}$ is not yet. Recall that, for positive operator $A$, the operator map $A \longmapsto A^{2}$ satisfies the following properties: $\left(o_{1}\right)$ the real-valued function $u \longmapsto q_{A}(u):=(1 / 2)\langle A u, u\rangle$ is convex,

$\left(\mathrm{o}_{2}\right)$ the map $A \longmapsto A^{2}$ is operator convex,

$\left(o_{3}\right)$ the map $A \longmapsto A^{2}$ is not operator monotone: $A \leq B$ does not ensure $A^{2} \leq B^{2}$, in general.

To extend $A \longmapsto A^{2}$ from positive operator to convex functional, we then search a functional map i.e. map involving functional arguments, denoted by $f \longmapsto f^{[2]}$, that satisfies the operator-functional connection, i.e.

(of) the functional-valued map $f \longmapsto f^{[2]}$ is really an extension of $A \longmapsto A^{2}$ via (1.1), with the next requirements:

$\left(f_{1}\right)$ the (extended) real-valued map $u \longmapsto f^{[2]}(u)$ is convex,

$\left(f_{2}\right)$ the functional-valued map $f \longmapsto f^{[2]}$ is point-wisely convex,

$\left(f_{3}\right)$ the functional-valued map $f \longmapsto f^{[2]}$ is in general not point-wisely monotone.

The fundamental goal of this paper is to give an affirmative answer to the above problem: we give an analogue of $A B A$ when the operator variables $A$ and $B$ are replaced by two convex functionals $f$ and $g$, respectively. As already pointed before, if this functional extension is denoted by $\Lambda(f, g)$ we should have the connection-relation $\Lambda\left(f_{A}, f_{B}\right)=f_{A B A}$, where $f_{A}$ is as above.

\section{BASIC NOTIONS}

In this section, we state some basic notions that will be needed throughout this paper. Let $H$ be a real or complex Hilbert space with the inner product $\langle.,$. and its associated norm $\|$.$\| . The (extended) space of all functions defined from$ $H$ into $\widetilde{\mathbb{R}}:=\mathbb{R} \cup\{\infty\}$ not identically equal to $\infty$ will be denoted here by $\widetilde{\mathbb{R}}^{H}$. This (extended) space will be endowed with the point-wise order defined by: if $f_{1}, f_{2} \in \widetilde{\mathbb{R}}^{H}$ we write $f_{1} \leq f_{2}$ if and only if $f_{1}(u) \leq f_{2}(u)$ for all $u \in H$, with the convention $x \leq \infty$ for all $x \in \mathbb{R} \cup\{\infty\}$. 
For a given $f \in \widetilde{\mathbb{R}}^{H}$, the Legendre-Fenchel conjugate of $f$ is the function $f^{*}$ defined by, [6]

$$
\forall u^{*} \in H \quad f^{*}\left(u^{*}\right)=\sup _{u \in H}\left\{R e\left\langle u, u^{*}\right\rangle-f(u)\right\},
$$

and its effective domain is dom $f=\{u \in H, \quad f(u)<\infty\}$. Obviously, $f^{*}(0)=$ $-\inf \{f(u), u \in H\}$ and if $f_{1} \leq f_{2}$ then $f_{2}^{*} \leq f_{1}^{*}$. The following

$$
\forall u, u^{*} \in H \quad R e\left\langle u, u^{*}\right\rangle \leq f(u)+f^{*}\left(u^{*}\right)
$$

is well-known as the Fenchel inequality in convex analysis.

We denote by $\sigma(u)=\frac{1}{2}\|u\|^{2}$ the unique self-conjugate function defined on $H$. The set of all convex (resp. convex and lower semi-continuous) functions defined from $H$ into $\widetilde{\mathbb{R}}$ not identically equal to $\infty$ is usually denoted by $\operatorname{Conv}(H)$ (resp. $\left.\Gamma_{\circ}(H)\right)$. It is well-known that if $f \in \operatorname{Conv}(H)$ then $f^{*} \in \Gamma_{\circ}(H)$ and, $f \in \Gamma_{\circ}(H)$ if and only if $f^{* *}:=\left(f^{*}\right)^{*}=f$.

The sub-differential of $f$ at $u \in H$ is the (possibly empty) convex closed subset of $H$ defined through

$$
\partial f(u)=\left\{u^{*} \in H ; \forall v \in H \quad f(v) \geq f(u)+R e\left\langle v-u, u^{*}\right\rangle\right\} .
$$

It is well-known that $u^{*} \in \partial f(u)$ if and only if $f(u)+f^{*}\left(u^{*}\right)=R e\left\langle u, u^{*}\right\rangle$ and, if $f \in \Gamma_{\circ}(H), u^{*} \in \partial f(u)$ if and only if $u \in \partial f^{*}\left(u^{*}\right)$.

For more details about the above notions in the case of real Hilbert, we refer the reader to [5, 7] for instance. The complex case can be stated in a similar manner.

For $f: H \longrightarrow \widetilde{\mathbb{R}}$ and $u, v \in H$, we set

$$
[u, v]_{f}=\lim _{t \downarrow 0^{+}} \frac{f(u+t . v)-f(u)}{t},
$$

provided that this limit exists in $[-\infty, \infty]$. In this case, $[u, v]_{f}$ which is nothing other than the directional derivative of $f$ is the real-part of the so-called semiinner product (or generalized inner product) of $u$ and $v$, with respect to $f$, defined through

$$
\forall u, v \in H \quad\langle u, v\rangle_{f}=[u, v]_{f}+i[u, i v]_{f} .
$$

If $f(u)=\sigma(u)$, the unique self-conjugate function, then $\langle u, v\rangle_{f}$ coincides with $\langle u, v\rangle$ the initial inner product of $H$, so justifying the terminology of generalized inner product.

An interesting situation is that where the functional $f$ is convex, [7]. In this case, for fixed $u, v \in H$, the function $\theta: t \longmapsto f(u+t . v)$ is convex and consequently the derivative $\theta^{\prime}(0)=[u, v]_{f}$ exists. In addition, the map

$$
] 0, \infty\left[\ni t \longmapsto \frac{f(u+t . v)-f(u)}{t}\right.
$$

is monotonically decreasing and so, for $f \in \operatorname{Conv}(H)$, we have

$$
[u, v]_{f}:=\lim _{t \downarrow 0^{+}} \frac{f(u+t . v)-f(u)}{t}=\inf _{t>0} \frac{f(u+t . v)-f(u)}{t} .
$$

The next example is of interest in the following. 
Example 2.1. Let $A$ be a (self-adjoint) positive invertible operator from $H$ into itself and take $f_{A}(u)=\frac{1}{2}\langle A u, u\rangle$ for every $u \in H$.

(i) It is well-known that $f_{A}^{*}\left(u^{*}\right)=\frac{1}{2}\left\langle A^{-1} u^{*}, u^{*}\right\rangle$ for each $u^{*} \in H$. We simply write $f_{A}^{*}=f_{A^{-1}}$. Otherwise, simple manipulation leads to $\partial f_{A}(u)=\{A u\}$ for all $u \in H$.

(ii) We can easily verify that $[u, v]_{f_{A}}=R e\langle A u, v\rangle$ for all $u, v \in H$. In particular, $[u, v]_{\sigma}=R e\langle u, v\rangle$. Remark that $f_{I}=\sigma$, where $I$ is the identity operator of $H$.

This example justifies again the terminology of generalized inner product with respect to $f$.

We end this section by stating the following result which will be needed later.

Proposition 2.2. Let $f \in \operatorname{Conv}(H)$ and $u \in H$ be fixed. Then the next assertions hold true.

(i) The map $\phi_{u}^{f}: v \longmapsto[u, v]_{f}$ is convex.

(ii) The conjugate of $\phi_{u}^{f}$ is given by

$$
\forall v^{*} \in H \quad\left(\phi_{u}^{f}\right)^{*}\left(v^{*}\right)=\Psi_{\partial f(u)}\left(v^{*}\right),
$$

where, for a subset $E$ of $H, \Psi_{E}$ refers to the indicator function of $E$ defined by $\Psi_{E}(u)=0$ if $u \in E$ and $\Psi(u)=\infty$ else.

Proof. (i) Since $f$ is convex and $v \longmapsto u+t v$ is linear affine with respect to $v$ then $v \longmapsto f(u+t v)$ is convex. It follows that

$$
H \ni v \longmapsto \frac{f(u+t v)-f(u)}{t},
$$

is convex and so is $v \longmapsto[u, v]_{f}$ as simple limit of a family of convex functionals. ii) For the case of real Hilbert, see [5]. The complex case may be stated in a similar manner. Detail is omitted here for the reader.

We notice that the above assertion (i) is among the interesting properties of the generalized inner product and extends the (anti) linearity of the inner product with respect to its second variable.

\section{ON A CONVEX FUNCTIONAL TRANSFORMATION}

As already pointed before, the aim of this section is to give an extension of $A B A$ when the operator variables $A$ and $B$ are convex functionals. We preserve the same notations as previous and we start by stating the following definition.

Definition 3.1. Let $f \in \operatorname{Conv}(H)$ and $g \in \widetilde{\mathbb{R}}^{H}$. We put

$$
\forall v \in H \quad \Lambda(f, g)(v):=\sup _{u \in H}\left\{[u, v]_{f}-g(u)\right\} .
$$

In particular, we set

$$
\forall v \in H \quad f^{[2]}(v):=\Lambda(f, \sigma)(v)=\sup _{u \in H}\left\{[u, v]_{f}-\sigma(u)\right\},
$$

which will be called the convex square of $f$. 
The elementary properties of the above functional transformation are stated in the following result.

Proposition 3.2. Let $f \in \operatorname{Conv}(H)$ and $g \in \widetilde{\mathbb{R}}^{H}$. Then the next assertions hold: (i) $\Lambda(\sigma, g)=g^{*}$, (extension of Legendre-Fenchel conjugacy).

(ii) For all $u, v \in H$ one has

$$
[u, v]_{f} \leq \Lambda(f, g)(v)+g(u), \quad \text { (extension of Fenchel inequality). }
$$

(iii) $\inf \{g(u), u \in d o m f\}=-\Lambda(f, g)(0)$, (characterization of the infimum).

(iv) Let $g_{1}, g_{2} \in \widetilde{\mathbb{R}}^{H}$. Then

$$
g_{1} \leq g_{2} \Longrightarrow \Lambda\left(f, g_{1}\right) \geq \Lambda\left(f, g_{2}\right), \text { (point-wise monotonicity). }
$$

(v) For all $\alpha>0$ we have

$$
\Lambda(\alpha . f, \alpha . g)=\alpha \cdot \Lambda(f, g), \quad \text { (joint-homogeneity). }
$$

(vi) The function $v \longmapsto \Lambda(f, g)(v)$ is convex. That is, for given $f \in \operatorname{Conv}(H)$, $\Lambda(f,$.$) is a map from Conv (H)$ into itself. In particular, $v \longmapsto f^{[2]}(v)$ is convex.

Proof. (i) Follows from (3.1) with (2.1) and Example 2.1,(ii).

(ii),(iii),(iv) and (v) are immediate from (3.1) with some convenient manipulations. Detail is simple and omitted here.

(vi) Following Proposition 2.2, the function $v \longmapsto[u, v]_{f}$ is convex and so is $v \longmapsto[u, v]_{f}-f(u)$, for fixed $u \in H$. It follows that $v \longmapsto \Lambda(f, g)(v)$ is convex as upper hull of a family of convex functionals. The proof of the proposition is completed.

Now, we are in position to state the most interesting result recited as follows.

Theorem 3.3. The map $(f, g) \longmapsto \Lambda(f, g)$ is point-wisely convex upon its functional variables $f$ and $g$. That is,

(1) Let $f \in \operatorname{Conv}(H)$ be fixed. Then the map $g \longmapsto \Lambda(f, g)$ is point-wise convex: for all $g_{1}, g_{2} \in \widetilde{\mathbb{R}}^{H}$ and $\left.\lambda \in\right] 0,1[$ one has

$$
\Lambda\left(f,(1-\lambda) \cdot g_{1}+\lambda \cdot g_{2}\right) \leq(1-\lambda) \cdot \Lambda\left(f, g_{1}\right)+\lambda \cdot \Lambda\left(f, g_{2}\right)
$$

(2) Let $g \in \widetilde{\mathbb{R}}$ be fixed. Then the mapping $f \longmapsto \Lambda(f, g)$ is point-wise convex: for all $f_{1}, f_{2} \in \operatorname{Conv}(H)$ and $\left.\lambda \in\right] 0,1[$ we have

$$
\Lambda\left((1-\lambda) \cdot f_{1}+\lambda \cdot f_{2}, g\right) \leq(1-\lambda) \cdot \Lambda\left(f_{1}, g\right)+\lambda \cdot \Lambda\left(f_{2}, g\right) .
$$

Proof. (1) It is clear that

$[u, v]_{f}-\left((1-\lambda) \cdot g_{1}+\lambda \cdot g_{2}\right)(u)=(1-\lambda) \cdot\left([u, v]_{f}-g_{1}(u)\right)+\lambda \cdot\left([u, v]_{f}-g_{2}(u)\right)$.

By (3.1), with the sub-additivity and the positive homogeneity of the "supremum", we obtain the desired inequality.

(2) By (2.2), it is easy to see that

$$
[u, v]_{(1-\lambda) \cdot f_{1}+\lambda \cdot f_{2}}=(1-\lambda) \cdot[u, v]_{f_{1}}+\lambda \cdot[u, v]_{f_{2}} .
$$

By the same arguments as above, we deduce the desired result. The proof of the theorem is completed. 
Theorem 3.4. The map $(f, g) \longmapsto \Lambda(f, g)$ is jointly super-additive and jointly point-wise convex. That is, for all $f_{1}, f_{2} \in \operatorname{Conv}(H), g_{1}, g_{2} \in \widetilde{\mathbb{R}}^{H}$ and $\left.\lambda \in\right] 0,1[$ there hold

$$
\begin{gathered}
\Lambda\left(f_{1}+f_{2}, g_{1}+g_{2}\right) \leq \Lambda\left(f_{1}, g_{1}\right)+\Lambda\left(f_{2}, g_{2}\right) \\
\Lambda\left((1-\lambda) \cdot f_{1}+\lambda \cdot f_{2},(1-\lambda) \cdot g_{1}+\lambda \cdot g_{2}\right) \leq(1-\lambda) \cdot \Lambda\left(f_{1}, g_{1}\right)+\lambda \cdot \Lambda\left(f_{2}, g_{2}\right) .
\end{gathered}
$$

Proof. We have

$$
[u, v]_{f_{1}+f_{2}}-\left(g_{1}+g_{2}\right)(u)=\left([u, v]_{f_{1}}-g_{1}(u)\right)+\left([u, v]_{f_{2}}-g_{2}(u)\right)
$$

from which the joint sub-additivity of $(f, g) \longmapsto \Lambda(f, g)$ follows in a similar manner as previous. This, when combined with the joint-homogeneity (Proposition $3.2,(\mathrm{v}))$, yields the joint point-wise convexity of $(f, g) \longmapsto \Lambda(f, g)$. The proof is complete.

Corollary 3.5. The following assertions hold true:

(i) Let $f \in \operatorname{Conv}(H)$. For all $u, v \in H$ one has

$$
[u, v]_{f} \leq \sigma(u)+f^{[2]}(v)
$$

(ii) Let $f \in \operatorname{Conv}(H)$. The functional $v \longmapsto f^{[2]}(v)$ is convex:

$\forall \lambda \in] 0,1\left[\quad \forall u, v \in H, \quad f^{[2]}((1-\lambda) u+\lambda v) \leq(1-\lambda) \cdot f^{[2]}(u)+\lambda \cdot f^{[2]}(v)\right.$.

(iii) The map $f \longmapsto f^{[2]}$ is point-wise convex, that is, the inequality

$$
\left((1-\lambda) \cdot f_{1}+\lambda \cdot f_{2}\right)^{[2]} \leq(1-\lambda) \cdot f_{1}^{[2]}+\lambda \cdot f_{2}^{[2]}
$$

holds for all $\lambda \in] 0,1\left[\right.$ and $f_{1}, f_{2} \in \operatorname{Conv}(H)$.

Proof. (i) Comes from (3.2) with Proposition 3.2,(ii).

(ii) Follows from Proposition 3.2,(vi), with (3.2).

(iii) It is immediate from Theorem 3.4,(2) with (3.2).

Now, we will state a result which justifies that the above functional transformation is really an extension of $A B A$ for convex functionals.

Theorem 3.6. Let $f=f_{A}$ and $g=f_{B}$, where $A$ and $B$ are two (self-adjoint) positive operators from $H$ into itself. Assume that further $B$ is invertible. Then we have

$$
\Lambda\left(f_{A}, f_{B}\right)=f_{C}, \text { with } C=A B^{-1} A
$$

In particular,

$$
\Lambda\left(f_{A}, \sigma\right)=f_{A^{2}} .
$$

Proof. By definition, with Example 2.1, we have

$$
\begin{aligned}
& \Lambda\left(f_{A}, f_{B}\right)(v)=\sup _{u \in H}\left\{[u, v]_{f_{A}}-f_{B}(u)\right\}=\sup _{u \in H}\left\{\operatorname{Re}\langle A u, v\rangle-\frac{1}{2}\langle B u, u\rangle\right\} \\
& =\sup _{u \in H}\left\{-\frac{1}{2}\left\|B^{1 / 2} u-B^{-1 / 2} A v\right\|^{2}+\frac{1}{2}\left\|B^{-1 / 2} A v\right\|^{2}\right\} \leq \frac{1}{2}\left\|B^{-1 / 2} A v\right\|^{2} .
\end{aligned}
$$


Further, this latter side is attained for $u=B^{-1} A v$ and so we have

$$
\begin{aligned}
\Lambda\left(f_{A}, f_{B}\right)(v)=\frac{1}{2}\left\|B^{-1 / 2} A v\right\|^{2}=\frac{1}{2}\left\langle B^{-1 / 2} A v\right. & \left.B^{-1 / 2} A v\right\rangle \\
& =\frac{1}{2}\left\langle A B^{-1} A v, v\right\rangle=f_{A B^{-1} A}(v) .
\end{aligned}
$$

The proof of the theorem is complete.

It is worth mentioning that, all the above results have been proved in a fast and simple way. This because the above transformation $(f, g) \longmapsto \Lambda(f, g)$ has a convex character. Some properties of operators can be immediately deduced by using the present functional approach. The following example explains this latter situation.

Example 3.7. Let $A$ and $B$ be as above. Combining Theorem 3.3, Theorem 3.4 and Theorem 3.6 we immediately deduce that the map $(A, B) \longmapsto A B^{-1} A$ is operator convex in $A$, in $B$ and jointly in $A$ and $B$. In particular, the maps $A \longmapsto A^{-1}$ and $A \longmapsto A^{2}$ are operator convex.

The following result, concerning the conjugate of the map $v \longmapsto \Lambda(f, g)(v)$, is of interest.

Theorem 3.8. Let $f \in \Gamma_{\circ}(H)$ and $g \in \widetilde{\mathbb{R}}^{H}$. Then the following inequality holds

$$
\forall v^{*} \in H \quad(\Lambda(f, g))^{*}\left(v^{*}\right) \leq \inf \left\{g(u), u \in \partial f^{*}\left(v^{*}\right)\right\} .
$$

Proof. By Proposition 3.2,(i), we have for all $u, v \in H$,

$$
\Lambda(f, g)(v) \geq[u, v]_{f}-g(u) .
$$

Taking the conjugates of the two sides, with respect to $v$, we deduce with help of Proposition 2.2,(ii)

$$
\forall v^{*} \in H \quad(\Lambda(f, g))^{*}\left(v^{*}\right) \leq \Psi_{\partial f(u)}\left(v^{*}\right)+g(u) .
$$

As pointed before, $v^{*} \in \partial f(u)$ if and only if $u \in \partial f^{*}\left(v^{*}\right)$ and the desired inequality follows after a simple manipulation.

Remark 3.9. With the assumptions of the above theorem, let $v^{*} \in H$ be such that $\partial f^{*}\left(v^{*}\right)$ is nonempty. If moreover $f \in \Gamma_{\circ}(H)$ then the "inf" in the second member of $(3.3)$ is attained, since $\partial f^{*}\left(v^{*}\right)$ is weakly compact and $g$ is weakly lower semi-continuous.

Finally, the next corollary may be stated.

Corollary 3.10. Let $f \in \Gamma_{\circ}(H)$. Then there holds

$$
\forall v^{*} \in H \quad\left(f^{[2]}\right)^{*}\left(v^{*}\right) \leq \frac{1}{2} d^{2}\left(0, \partial f^{*}\left(v^{*}\right)\right),
$$

where, for $E \subset H$, the notation $d(0, E)$ refers to the distance from 0 to $E$ : $d(0, E):=\inf _{u \in E}\|u\|$ with the convention $d(0, \emptyset)=\infty$. 
Proof. By (3.3) we have

$$
\left(f^{[2]}\right)^{*}\left(v^{*}\right) \leq \inf \left\{\sigma(u), u \in \partial f^{*}\left(v^{*}\right)\right\}=\frac{1}{2}\left(\inf \left\{\|u\|, u \in \partial f^{*}\left(v^{*}\right)\right\}\right)^{2}
$$

from which the desired inequality follows.

Acknowledgement. Many thanks to the anonymous referee for valuables comments and suggestions that have been included in the final version of this manuscript.

\section{REFERENCES}

1. M. Atteia and M. Raïssouli, Self dual operator on convex functionals, geometric mean and square root of convex functionals, J. Convex Anal. 8 (2001), no. 1, 223-240.

2. J.I. Fujii, Kubo-Ando theory for convex functional means, Sci. Math. Jpn. 7 (2002), no. 2, 299-311.

3. J.A. Johnstone, V.R. Koch and Y. Lucet, Convexity of the proximal average, J. Optim. Theory Appl. 148 (2011), no. 1, 107-124.

4. S. Kum and Y. Lim, A geometric mean of parameteized arithmetic and harmonic means of convex functions, Abst. Appl. Anal. 2012, Art. 836804, 15 pages.

5. P.J. Laurent, Approximation et optimisation, Hermann, 1972.

6. M. Raïssouli, Extension of shorted operator for convex functionals, Ann. Sci. Math. Québec 36 (2012), no. 2, 179-192.

7. E. Zeidler, Nonlinear functional analysis and its applications III, Springer-Verlag, 1984.

Department of Mathematics, Science Faculty, Taibah University, Al Madinah Al Munawwarah, P.O.Box 30097, Zip Code 41477, Saudi Arabia.

Department of Mathematics, Science Faculty, Moulay Ismail University, MekNES, MOROCCO.

E-mail address: raissouli_10@hotmail.com 